\title{
Consumo de alcohol y los roles del bullying en adolescentes
}

\author{
Henry Amaro-Berríos ${ }^{1}$ \\ Vanessa Azaña-Velezmoro ${ }^{2}$
}

RESUMEN: Objetivo: Explicar los efectos del consumo de alcohol sobre el bullying en un grupo de estudiantes del nivel secundario en Lima y Callao. Materiales y métodos: Se realizó un análisis secundario de la base de datos recogida en el IV Estudio Nacional: Prevención y Consumo de Drogas en Estudiantes de Secundaria - 2012, a cargo de DEVIDA. La muestra fue de 6 125 estudiantes entre los 11 y 20 años de edad que cursaban del 1ro al 5to de secundaria en colegios públicos y privados en Lima y Callao. La variable dependiente fue el bullying considerando sus roles: exposición, victimización y agresividad. La variable independiente fue el consumo de alcohol. Se calcularon Odds ratios (OR) para estimar la fuerza de las asociaciones entre las variables mediante regresión ordinal. Resultados: El análisis multivariado reportó un aumento de la exposición al bullying asociado al consumo de alcohol en el último mes (OR=1.52; IC 95\%: 1.26-1.82) y un aumento del ejercer agresión a través del bullying $(\mathrm{OR}=1.50$; IC 95\%: 1.24-1.82). Conclusiones: existe una asociación significativa entre el beber alcohol y los diferentes roles del bullying. Sin embargo, el consumo de alcohol expone mayormente asumir el rol de agresor, igualmente el ser varón. La presencia de algún tipo de discapacidad se asocia al incremento de bullying en sus tres roles.

PALABRAS CLAVE: Citar como:
Consumo de alcohol en menores; Acoso escolar; Adolescente.

Amaro H, Azaña V. Consumo de alcohol y su relación con los roles del bullying en adolescentes. CASUS. 2017;2(1):28-36. 


\section{INTRODUCCIÓN}

El consumo de alcohol y el bullying son dos comportamientos problemáticos que prevalecen en la adolescencia. Ambos fenómenos cuentan con desencadenantes personales, familiares y sociales (1-3). Diversos estudios señalan que ambas conductas se encuentran asociadas en esta etapa (4-9).

En la adolescencia el consumo de alcohol es un factor de riesgo para la salud ya que genera daños a nivel físico y mental $(5,9)$, además de una mayor vulnerabilidad psicosocial (1). Estudios señalan su relación con un bajo rendimiento académico (1), abandono escolar (10) y problemas de salud mental como síntomas psicosomáticos y depresivos $(5,6,11$, 12). Asimismo, existen reportes que lo asocian con el incremento de conductas agresivas y violentas $(6,13$, 14). A nivel nacional el 40.5\% (900 000 estudiantes) de la población escolar señala haber consumido alcohol alguna vez en su vida, mientras que la prevalencia anual del consumo de drogas legales (incluidas el alcohol y el tabaco) para los estudiantes de Lima Metropolitana es de $26.4 \%$ y para el Callao de $33.4 \%$ (15).

El fenómeno del bullying entendido como el acoso planificado y sistemático hacia una persona en el ámbito escolar (16) está relacionado a afectaciones psicosociales. Entre ellas destacan alteraciones intensas del estado afectivo y del carácter, deterioro de la autoestima, el aislamiento familiar y social (1, 11, 16). Las mismas generan limitaciones significativas en las relaciones interpersonales, en el rendimiento académico vinculándose a conductas asociadas al consumo de sustancias $(3,4,11,17,18)$. Estos datos son reportados en algunos estudios peruanos $(7,15)$. A nivel nacional, el bullying es una situación problemática muy frecuente en el contexto escolar. Se estima que el $47.5 \%$ de la población de escolares ha sido agredida en la escuela en el último mes. Mientras que un $38 \%$ ha sido víctima de agresiones físicas en los últimos doce meses (19).

Numerosos antecedentes coinciden en que el consumo de alcohol y el bullying están asociados (7-9). El consumo de alcohol incrementa la exposición a conductas de riesgo y de violencia en los adolescentes $(4,6,20)$. De esta manera el beber puede relacionarse con la imagen del consumidor frente a los demás ya sea para obtener respeto, aceptación o un lugar dentro del grupo. Así mismo, el uso de alcohol en adolescentes se asocia al desarrollo de conductas agresivas que se vinculan al bullying (4-7). Los estudiantes que presentan un patrón personal de comportamiento agresivo serían potenciales agresores a través del bullying $(4,20)$. Además, los adolescentes consumidores de alcohol se encuentran involucrados en actos de violencia en cualquiera de sus roles. Los mismos están propensos a desarrollar comportamientos que refuerzan la idea de rudeza hacía sus pares con el objetivo de ser respetados (17, 20, 21). El contexto escolar es un entorno en que ambos fenómenos pueden coincidir y los alumnos pueden verse involucrados como agresores, víctimas u observadores.

No obstante ha sido bien documentada la alta prevalencia de consumo de alcohol y bullying entre los adolescentes (15) existe poca información sobre su relación con los diferentes roles del bullying. Es necesario conocer con mayor precisión los efectos de este consumo sobre los roles del bullying en los escolares para poder plantear estrategias de evaluación temprana del abuso de esta sustancia en la realización de programas de prevención e intervención del bullying entre adolescentes. De esta manera se propuso como objetivo explicar los efectos del consumo de alcohol sobre el bullying en estudiantes de Lima.

\section{MATERIALES Y MÉTODOS}

Se realizó un análisis secundario de la base de datos recogida en el IV Estudio Nacional: Prevención y Consumo de Drogas en Estudiantes de Secundaria 2012, a cargo de DEVIDA. El muestreo fue probabilístico, bietápico y estratificado, dependiendo de la gestión del colegio (público o privado) y de la región, teniendo como unidad primaria de muestreo a los colegios y como unidad secundaria a las aulas seleccionadas aleatoriamente. La muestra fue de 6125 estudiantes entre los 11 y 20 años de edad que cursaban del 1ro al 5to de secundaria en colegios en Lima y Callao. Para la recolección de datos se empleó un instrumento auto aplicado, diseñado por DEVIDA, de 138 preguntas de opción múltiple y con un registro de respuestas en hojas ópticas. El mismo se creó en base a un instrumento estandarizado propuesto por la Comisión Interamericana para el Control de Abuso de Drogas (CICAD) y el Sistema Interamericano de Datos Uniformes sobre Consumo de Drogas (SIDUC).

La variable dependiente fue el bullying definida como la violencia repetitiva y sistemática generada entre 
pares de un mismo colegio existiendo una intencionalidad por parte del agresor y un desequilibrio de poderes entre agresor y víctima (3, 11, 16). Para medir esta variable en la encuesta se emplearon tres escalas orientadas a: la exposición al bullying (testigo de la violencia o exposición indirecta); la victimización por bullying (exposición directa); y la agresividad a través del bullying (rol de agresor). Categorizándose en nivel bajo, medio y alto, respectivamente. La variable independiente fue el consumo de alcohol y se originó en base a la pregunta: ¿Has consumido alcohol en los últimos treinta días?, y se categorizó como: sí y no.

Las variables sociodemográficas consideradas fueron: sexo, variable dicotómica (masculino y femenino); edad, variable continua; tipo de colegio, variable dicotómica (público y privado); lugar de residencia, dicotómica (Lima Metropolitana y Callao); año de estudio, variable politómica (primero, segundo, tercero, cuarto y quinto). Las variables de ajuste para el análisis multivariado fueron: consumo de tabaco, y se originó en base a la pregunta: ¿Has consumido cigarrillos en los últimos treinta días?, variable dicotómica (sí y no); consumo de drogas ilegales, que se originó en base a la pregunta: ¿Has consumido alguna droga ilegal en los últimos treinta días?, variable dicotómica (sí y no) y discapacidad, la cual se originó en base a los siguientes criterios: usa lentes, no escucha bien, cojea, dificultad para hablar, problemas de aprendizaje (definida como una limitación en la función al momento de la toma de la encuesta), variable dicotómica (sí y no).
Para analizar la base de datos se utilizó el programa estadístico STATA versión 13. Se utilizó la prueba de Kolmogorrov Smirnov para determinar la normalidad de las variables y realizar las pruebas estadísticas de Chi-cuadrado, ANOVA y la prueba t. Se tomó en cuenta las asociaciones en el análisis bivariado para realizar la regresión logística ordinal. Se calcularon Odds Ratio (OR) y se realizó el análisis multivariado para obtener el OR crudo y ajustado. Para el análisis inferencial se usó un nivel de confianza al 95\% y un valor de $\mathrm{p}<0.05$.

En la recolección de los datos DEVIDA respetó la identidad de los estudiantes considerando los criterios éticos de confidencialidad en las respuestas y el anonimato de los encuestados. La presente investigación fue aprobada por el Comité de Ética de la Universidad Católica Sedes Sapientiae.

\section{RESULTADOS}

Respecto a la muestra de estudio el $52.16 \%$ fueron mujeres con un promedio de edad de 14.26 años. El $59.76 \%$ de los encuestados provenía de colegios públicos y el $22.01 \%$ cursaba el segundo año de educación secundaria. E1 38.43\% de los encuestados presentó alguna discapacidad. El $48.58 \%$ de los alumnos estuvo expuesto al bullying en un nivel alto, el $63.52 \%$ indicó un nivel bajo de victimización, mientras que el $67.02 \%$ reportó un nivel bajo de agresión. La co-ocurrencia de consumo de alcohol y bullying fue de un $11.9 \%$. En el último mes, el $11.44 \%$ de los estudiantes consumió alcohol y tabaco $(8.59 \%)$ (ver tabla 1).

Tabla 1. Características de estudiantes del nivel secundario en Lima Metropolitana y Callao ( $n=6$ 125)

\begin{tabular}{llll}
\hline Factores & & $\mathbf{N}$ & $\%$ \\
\hline Sexo & Masculino & 2930 & 47.84 \\
Edad (media \pm DS) & Femenino & 3195 & 52.16 \\
Tipo de colegio & & & $14.26 \pm 1.53$ \\
& Público & 3660 & 59.76 \\
Lugar de residencia & Privado & 2465 & 40.24 \\
& Lima Metropolitana & 4150 & 67.76 \\
Año de estudio & Callao & 1975 & 32.24 \\
& Primero & 1293 & 21.11 \\
& Segundo & 1348 & 22.01 \\
& Tercero & 1204 & 19.66 \\
& Cuarto & 1181 & 19.28 \\
& Quinto & 1099 & 17.94
\end{tabular}


Tabla 1. (continúa)

\begin{tabular}{llrr}
\hline Factores & & $\mathbf{N}$ & $\%$ \\
\hline Discapacidad & No & 3771 & 61.57 \\
& Sí & 2354 & 38.43 \\
Exposición al bullying & Nivel bajo & 810 & 13.73 \\
& Nivel medio & 2224 & 37.69 \\
& Nivel alto & 2867 & 48.58 \\
Victimización por bullying & Nivel bajo & 3835 & 63.52 \\
& Nivel medio & 1722 & 28.52 \\
Agresividad a través del bullying & Nivel alto & 480 & 7.95 \\
& Nivel bajo & 3832 & 67.02 \\
& Nivel medio & 1512 & 26.44 \\
Consumo de alcohol & Nivel alto & 374 & 6.54 \\
& No & 5424 & 88.56 \\
Consumo de tabaco & Sí & 701 & 11.44 \\
& No & 5599 & 91.41 \\
Consumo de drogas ilegales & Sí & 526 & 8.59 \\
& No & 6113 & 99.80 \\
& Sí & 12 & 0.20 \\
\hline
\end{tabular}

El consumo de alcohol en el último mes se asocia con un aumento de la exposición al bullying respecto a los que no consumen. Igualmente pertenecer al quinto grado de secundaria aumenta dicha exposición. El consumo de drogas ilegales y el consumo de tabaco incrementan dicho componente del bullying (ver tabla 2).

Tabla 2. Análisis multivariado del consumo de alcohol y la exposición al bullying

\begin{tabular}{llrrrrrr}
\hline Variables & & \multicolumn{3}{c}{ Modelo Crudo } & \multicolumn{3}{c}{ Modelo Ajustado } \\
& & OR & IC 95\% & P & OR & IC 95\% & P \\
\hline Consumo de alcohol & Sí & 1.91 & $(1.63-2.25)$ & $<0.001$ & 1.52 & $(1.26-1.82)$ & $<0.001$ \\
Consumo de tabaco & Sí & 1.93 & $(1.60-2.33)$ & $<0.001$ & 1.37 & $(1.10-1.69)$ & 0.004 \\
Consumo drogas ilegales & Sí & 2.08 & $(1.48-2.92)$ & $<0.001$ & 1.51 & $(1.06-2.16)$ & 0.023 \\
Discapacidad & Sí & 1.16 & $(1.05-1.29)$ & 0.003 & 1.17 & $(1.05-1.29)$ & 0.003 \\
Sexo & Femenino & 0.79 & $(0.72-0.88)$ & $<0.001$ & 0.80 & $(0.72-0.88)$ & $<0.001$ \\
Año de estudio & Segundo & 1.27 & $(1.09-1.47)$ & 0.002 & 1.31 & $(1.12-1.54)$ & 0.001 \\
& Tercero & 1.34 & $(1.15-1.56)$ & $<0.001$ & 1.42 & $(1.16-1.75)$ & 0.001 \\
& Cuarto & 1.48 & $(1.27-1.72)$ & $<0.001$ & 1.59 & $(1.23-2.06)$ & $<0.001$ \\
Edad & Quinto & 1.67 & $(1.43-1.96)$ & $<0.001$ & 1.86 & $(1.36-2.53)$ & $<0.001$ \\
& & 1.09 & $(1.06-1.13)$ & $<0.001$ & 0.94 & $(0.88-1.01)$ & 0.106 \\
\hline
\end{tabular}


El haber consumido tabaco en el último mes se asocia con un aumento de los niveles de victimización por bullying. El uso de drogas ilegales y la presencia de alguna discapacidad aumentan los niveles de victimización por bullying (tabla 3 ).

Tabla 3. Análisis multivariado del consumo de alcohol y la victimización por bullying

\begin{tabular}{llcccccc}
\hline Variables & & \multicolumn{3}{c}{ Modelo Crudo } & \multicolumn{3}{c}{ Modelo Ajustado } \\
& & OR & IC 95\% & P & OR & IC 95\% & P \\
\hline Consumo de alcohol & Sí & 1.36 & $(1.16-1.60)$ & $<0.001$ & 1.10 & $(0.91-1.33)$ & 0.304 \\
Consumo de tabaco & Sí & 1.60 & $(1.34-1.91)$ & $<0.001$ & 1.30 & $(1.06-1.61)$ & 0.013 \\
Consumo drogas ilegales & Sí & 2.39 & $(1.75-3.28)$ & $<0.001$ & 1.96 & $(1.41-2.72)$ & $<0.001$ \\
Discapacidad & Sí & 1.41 & $(1.27-1.56)$ & $<0.001$ & 1.48 & $(1.28-1.59)$ & $<0.001$ \\
Sexo & Femenino & 0.70 & $(0.63-0.77)$ & $<0.001$ & 0.69 & $(0.62-0.77)$ & $<0.001$ \\
Año de estudio & Segundo & 1.12 & $(0.95-1.31)$ & 0.165 & 1.08 & $(0.90-1.29)$ & 0.393 \\
& Tercero & 1.11 & $(0.94-1.30)$ & 0.223 & 1.04 & $(0.83-1.30)$ & 0.715 \\
& Cuarto & 1.25 & $(1.06-1.47)$ & 0.008 & 1.14 & $(0.87-1.49)$ & 0.345 \\
Edad & Quinto & 1.15 & $(0.97-1.36)$ & 0.110 & 1.02 & $(0.73-1.41)$ & 0.917 \\
& & 1.04 & $(1.01-1.08)$ & 0.018 & 1.02 & $(0.94-1.09)$ & 0.687 \\
\hline
\end{tabular}

El consumo de alcohol se asocia asimismo con un aumento de los niveles del ser agresor a través del bullying. El análisis multivariado indica un efecto mayor del consumo de alcohol sobre el rol de agresor respecto a la victimización y la exposición a través del bullying. Del mismo modo, el consumo de tabaco, de drogas ilegales y presentar alguna discapacidad aumentan dicho rol (tabla 4).

Tabla 4. Análisis multivariado del consumo de alcohol y la agresividad a través del bullying

\begin{tabular}{llcccccc}
\hline Variables & & \multicolumn{3}{c}{ Modelo Crudo } & \multicolumn{4}{c}{ Modelo Ajustado } \\
& & OR & IC 95\% & P & OR & IC 95\% & P \\
\hline Consumo de alcohol & Sí & 2.21 & $(1.89-2.60)$ & $<0.001$ & 1.50 & $(1.24-1.82)$ & $<0.001$ \\
Consumo de tabaco & Sí & 2.78 & $(2.32-3.33)$ & $<0.001$ & 1.74 & $(1.41-2.16)$ & $<0.001$ \\
Consumo drogas ilegales & Sí & 4.33 & $(3.16-5.93)$ & $<0.001$ & 2.57 & $(1.84-3.61)$ & $<0.001$ \\
Discapacidad & Sí & 1.20 & $(1.07-1.34)$ & 0.002 & 1.24 & $(1.11-1.39)$ & $<0.001$ \\
Sexo & Femenino & 0.43 & $(0.38-0.48)$ & $<0.001$ & 0.43 & $(0.39-0.48)$ & $<0.001$ \\
Año de estudio & Segundo & 1.20 & $(1.01-1.42)$ & 0.043 & 1.10 & $(0.91-1.33)$ & 0.315 \\
& Tercero & 1.24 & $(1.04-1.48)$ & 0.016 & 1.04 & $(0.82-1.32)$ & 0.722 \\
& Cuarto & 1.60 & $(1.35-1.91)$ & $<0.001$ & 1.24 & $(0.93-1.66)$ & 0.139 \\
Edad & Quinto & 1.42 & $(1.89-1.69)$ & $<0.001$ & 0.99 & $(0.70-1.40)$ & 0.935 \\
& & 1.11 & $(1.07-1.15)$ & $<0.001$ & 1.05 & $(0.98-0.14)$ & 0.184 \\
\hline
\end{tabular}




\section{DISCUSIÓN}

El estudio examinó los efectos del consumo de alcohol sobre el bullying en un grupo de estudiantes del nivel secundario en Lima y Callao. Los resultados revelan la existencia de relación entre el consumo de alcohol y el bullying. Una asociación significativa entre el beber alcohol y los diferentes roles del bullying emerge de forma consistente principalmente con el rol de agresor.

Se evidenció claramente que el consumo de alcohol se asocia positivamente con el rol de agresor. El consumo de alcohol incrementa las conductas agresivas relacionadas al bullying pudiendo tener distintas causales. Entre ellas el buscar aminorar la carga emocional por la que atraviesa el estudiante, un mejor posicionamiento dentro del grupo de pares y el incrementar los niveles de seguridad (22). Asimismo, esta asociación positiva puede estar relacionada al deseo de los agresores de ganar estatus social y ser percibidos como más atractivos. Durante la adolescencia, el beber suele ser un comportamiento que contribuye a la imagen social del individuo dentro del grupo de pares (23). Por otro lado, existe consenso al afirmar que el consumo de alcohol en adolescentes se asocia al desarrollo de conductas agresivas, como el caso del bullying $(4,5,7,14,17)$. Los estudiantes que presentan un patrón de comportamiento agresivo podrían convertirse en futuros agresores a través del bullying por su relación con conductas catalogadas como disociales causadas por el consumo de alcohol $(4,20)$. Además, los adolescentes consumidores de alcohol se encuentran involucrados en actos de violencia, ya sea de forma individual o grupal, y están propensos a desarrollar comportamientos que refuerzan la idea de rudeza hacía sus pares con el objetivo de ser respetados. El hecho de consumir alcohol en grupo podría ejercer una presión en los estudiantes, haciendo que los niveles de agresividad se incrementen, a diferencia de aquellos que no son consumidores de alcohol (14, 17).

Se encontró, asimismo, que el consumo de alcohol se asocia con el incremento de los niveles de victimización por bullying. Lo que concuerda con lo reportado en otros estudios donde se indicó que existen mayores probabilidades de ser víctimas de bullying entre los estudiantes consumidores de alcohol durante el último mes $(5,8,24,25)$. En este sentido, los resultados extienden la investigación anterior mostrando que el uso de alcohol aumenta la victimización en una muestra representativa de adolescentes de Lima.

El consumo de alcohol durante el último mes se asocia con el incremento en la exposición al bullying. Estudios previos indican que existe un alto nivel de consumo de sustancias, entre ellas el alcohol, en aquellos estudiantes expuestos al bullying (6). El consumo de alcohol predijo un riesgo de ser agresor, víctima o ser testigo del fenómeno. Este dato sugiere que tanto las víctimas como los observadores de bullying pueden compartir aspectos con los agresores (6). Entre ellos el beber alcohol como comportamiento recurrente en el grupo de pares con la finalidad de ganar aceptación y respeto dentro del mismo. Otro factor común pudiera ser la afiliación con pares que presentan problemas de conducta. Esta se encuentra correlacionada a una amplia gama de comportamientos nocivos incluyendo el uso de sustancias y las conductas de intimidación (25).

En relación a la presencia de discapacidad se incrementan los niveles de bullying en cualquiera de sus roles. De igual modo, presentar discapacidad influye directamente en el incremento de verse involucrado en los roles del bullying, siendo más fuerte su asociación con el rol de víctima. El considerar que se tiene una discapacidad se conecta con características que podrían acarrear dificultades en la relación entre pares predisponiendo a la victimización por bullying (26). Estos resultados concuerdan con estudios realizados en el país donde se reporta que los estudiantes con alguna discapacidad tienen un riesgo tres veces mayor hacia el bullying (27). Por otro lado, en los agresores el presentar alguna discapacidad incrementa las conductas de agresión a través del bullying. En las víctimas de bullying en casi el doble frente a los agresores. Este reporte concuerda también con estudios latinoamericanos (26).

El ser varón incrementa la presencia de bullying en cualquiera de sus roles respecto al ser mujer. Sin embargo, se asocia en modo más fuerte a la agresión a través del bullying. Esto concuerda con gran parte de la literatura $(5,17,28)$. No obstante, existe evidencia de que en los consumidores de alcohol de ambos sexos se presentan conductas de agresividad (17). En general, comparado con las estudiantes, las agresiones físicas y directas son aquellas que suelen tener mayor presencia en varones (5). Son los varones consumidores de alcohol quienes están más 
implicados en agresiones graves o severas $(5,17,28)$. Por otra parte, considerando los resultados, el ser mujer y no consumir alcohol, podría actuar como factor protector frente a los roles del bullying. Este dato es también soportado por estudios previos $(9,17$, 28).

El consumo de drogas ilegales y tabaco se asocia al aumento en la presencia de bullying en sus tres roles. Principalmente en el caso del consumo de drogas ilegales se relaciona a un incremento de las conductas de agresión y de victimización. Esto concuerda con lo reportado por la literatura, donde el consumo de sustancias psicoactivas aumenta el riesgo de ser agresor $(7,24,29-31)$.

Los resultados del estudio se deben interpretar en base a las siguientes limitaciones. La base de datos utilizada no presenta información relativa a otras variables de interés que pudieran ayudar en la comprensión del fenómeno. En este sentido, si bien el análisis comprende una serie de variables sociodemográficas, sin embargo, es importante considerar en futuros estudios otras variables confusoras vinculadas al rol parental de control y a la relación con los pares. La veracidad de la información obtenida por medio de las escalas de auto reporte podría haber sido condicionada por la deseabilidad social.

\section{CONCLUSIONES}

El consumo de alcohol y el bullying son comportamientos prevalentes en los adolescentes estudiados vinculados a una amplia gama de consecuencias adversas para la salud. Ambas situaciones son sociales y pueden coexistir como parte de la interacción en el grupo de adolescentes. Existe una asociación significativa entre el beber alcohol y los diferentes roles del bullying. Sin embargo, el consumo de alcohol expone mayormente al rol de agresor, igualmente el ser varón. La presencia de algún tipo de discapacidad se asocia al incremento de bullying en sus tres roles. Aunque en los adolescentes con discapacidad predomina la victimización. Por último, el consumo de drogas ilegales y tabaco se asocian con el aumento en los niveles de victimización y agresividad.

En el estudio se hipotetizó que los adolescentes que consumieron alcohol estarían mayormente predispuestos a estar involucrados en el bullying. Sin embargo, otros estudios reportan también la relación inversa de causalidad. La asociación positiva y significativa entre consumo de alcohol y la victimización por bullying subraya la necesidad de intervenir con los comportamientos de intimidación entre los adolescentes. En este sentido, es necesario el desarrollo de programas de intervención psicológica a nivel primario en la población adolescente y sus familias, para disminuir la exposición a los factores asociados al consumo de alcohol y al bullying. Los cuales incidiendo en la educación de padres de familia deben concientizar en el riesgo personal del consumo de alcohol y en las repercusiones negativas del bullying.

\section{REFERENCIAS BIBLIOGRÁFICAS}

1. Caña M, Michelini $\mathrm{Y}$, Acuña I, Godoy J. Efectos de la impulsividad y el consumo de alcohol sobre la toma de decisiones en los adolescentes. Salud y Drogas. 2015; 1(15):55-66.

2.

Jackson C, Ennett S, Dickinson D, Bowling J. Letting children sip: Understanding why parents allow alcohol use by elementary school-aged children. Arch Pediatr Adolesc Med. 2012;166(11):1053-7.

3. Tharp-Taylor S, Haviland A, D'Amico J. Victimization from Mental and Physical Bullying and Substance Use in Early Adolescence. Addictive behaviors. 2009; 34(6/7):561-7.
4. Cerezo F, Méndez I. Agresores en bullying y conductas antisociales. European Journal of Investigation in Health, Psychology and Education. 2013; 3(1):5-14.

5. Nansel T, Overpeck M, Pilla R, June W, Simons-Morton B, Scheidt P. Bullying Behaviors Among US Youth: Prevalence and Association with Psychosocial Adjustment. Year Book of Psychiatry \& Applied Mental Health. 2003; 285(16):2094-100.

6. Rivers I, Poteat V, Noret N, Ashurst N. Observing bullying at school: The mental health implications of witness status. School Psychology Quarterly. 2009; 24(4):211-23.
7. Romaní F, Gutiérrez C, Lama M. Auto-reporte de agresividad escolar y factores asociados en escolares peruanos de educación secundaria. Revista Peruana de Epidemiología. 2011; 15(2):118-25.

8. Vieno A, Gini G, Santinello $M$. Different forms of bullying and their association to smoking and drinking behavior in Italian adolescents. Journal of School Health. 2011; 81(7):393-9.

9. Méndez I, Cerezo F. Bullying y factores de riesgo para la salud en estudiantes de secundaria. European Journal of Education and Psychology. 2010; 3(2):209-18. 
10. García L, Orellana O, Yanac E, Herrera, et al. Factores predictores de la intimidación entre iguales desde la percepción de docentes y estudiantes de educación secundaria. Revista de Investigación en Psicología. 2013; 16(1):13-27.

11. Olweus, D. Conductas de acoso y amenaza entre escolares. $1^{\mathrm{a}}$ ed. Madrid, España: Ediciones Morata; 1993.

12. López-Caneda E, Rodríguez Holguín S, Cadaveira F, Corral M, Doallo S. Impact of Alcohol Use on Inhibitory Control (and Vice Versa) During Adolescence and Young Adulthood. Review Alcohol and Alcoholism. 2013; 49(2):173-81.

13. Becoña, E. Los adolescentes y el consumo de drogas. Papeles del psicólogo. 2000; (77):25-32.

14. Kuntsche E, Knibbe R, Engels R, Gmel G. Drinking motives as mediators of the link between alcohol expectancies and alcohol use among adolescents. Journal of Studies on Alcohol and Drugs. 2007 68(1):76-85.

15. Comisión Nacional para el Desarrollo y Vida sin Drogas (DEVIDA) [internet]. Lima, Perú: Comisión Nacional para el Desarrollo y Vida sin Drogas, Observatorio Peruano de Drogas; 2013 [citado el 15 enero de 2017]. Disponible

en: http://www.devida.gob.pe/2013/07/i nforme-ejecutivo-iv-estudio-naciona 1-prevencion-y-consumo-de-drogas-e n-estudiantes-de-secundaria-2012/

16. Olweus D. Acoso escolar, "bullying", en las escuelas: hechos e intervenciones. Centro de Investigación para la Promoción de la Salud, Universidad de Bergen, Noruega; 1993.
17. Moñino $\mathrm{M}$, Piñero $\mathrm{E}$, Arense $\mathrm{J}$, Cerezo F. Violencia escolar y consumo de alcohol y tabaco en estudiantes de Educación Secundaria. European Journal of Investigation in Health, Psychology and Education. 2013; 2(3):137-47.

18. Plan - UNICEF. Violencia escolar en América Latina y el Caribe: Superficie y fondo [internet]. Panamá: UNICEF; 2011 [citado el 15 enero de 2017]. Disponible en http://www.unicef.org/lac/violencia_e scolar_OK.pdf

19. Ministerio de Salud, Dirección General de Promoción de la Salud. Encuesta Global de Salud Escolar: resultados - Perú 2010. Lima: Ministerio de Salud; 2011.

20. Cerezo F. Diferencias en estatus social entre roles en bullying: Un análisis sociométrico. Bordón. 2014; 66(2):33-46.

21. Esteves E. Violencia, victimización y rechazo escolar en la adolescencia [Tesis doctoral]. Valencia: Servei De Publicacions; 2005. Disponible en: https://www.uv.es/lisis/estevez/tesises tevez.pdf

22. Bandura A, Walters R. Aprendizaje social de la conducta de dependencia. $7^{\text {a }}$ ed. Madrid: Alianza Editorial; 1974.

23. Ioannou S. Young people's accounts of smoking, exercising, eating and drinking alcohol: being cool or unhealthy. Journal Critical Public Health. 2003; (13):357-71.

24. Luk J, Wang J, Simons-Morton B. Bullying victimization and substance use among US adolescents: Mediation by depression. Prevention Science. 2010; 11(4):355-9.
25. Luk J, Wang J, Simons-Morton, B. The co-occurrence of substance use and bullying behaviors among US adolescents: Understanding demographic characteristics and social influences. Journal of adolescence. 2012; 35(5):1351-60.

26. Joffre-Velázquez V, García-Maldonado G, Saldívar-Gonzáles, et al. Bullying en alumnos de secundaria. Características generales y factores asociados al riesgo. Bol Med Hosp Infant Mex. 2011; 68(3):193-202.

27. Amemiya I, Oliveros M, Barrientos A. Factores de riesgo de violencia escolar (bullying) severa en colegios privados de tres zonas de la sierra del Perú. En Anales de la Facultad de Medicina, UNMSM. 2009; 70(4):255-8.

28. Benítez J, Justicia F. El maltrato entre iguales: descripción y análisis del fenómeno. Revista Electrónica de Investigación Psicoeducativa. 2006; 4(2):151-70.

29. Radcliff K, Wheaton J, Robinson K, Morris J. Illuminating the relationship between bullying and substance use among middle and high school youth. Addictive Behaviors. 2012; 37(4):569-72.

30. Archimi A, Kuntsche E. Do offenders and victims drink for different reasons? Testing mediation of drinking motives in the link between bullying subgroups and alcohol use in adolescence. Addictive Behaviors. 2014; 39(3):713-6.

31. Fleming L, Jacobsen K. Bullying among middle-school students in low and middle-income countries. Health Promotion International. 2009; 25(1):73-84. 


\section{Alcohol use and its relation with the roles of bullying in adolescents}

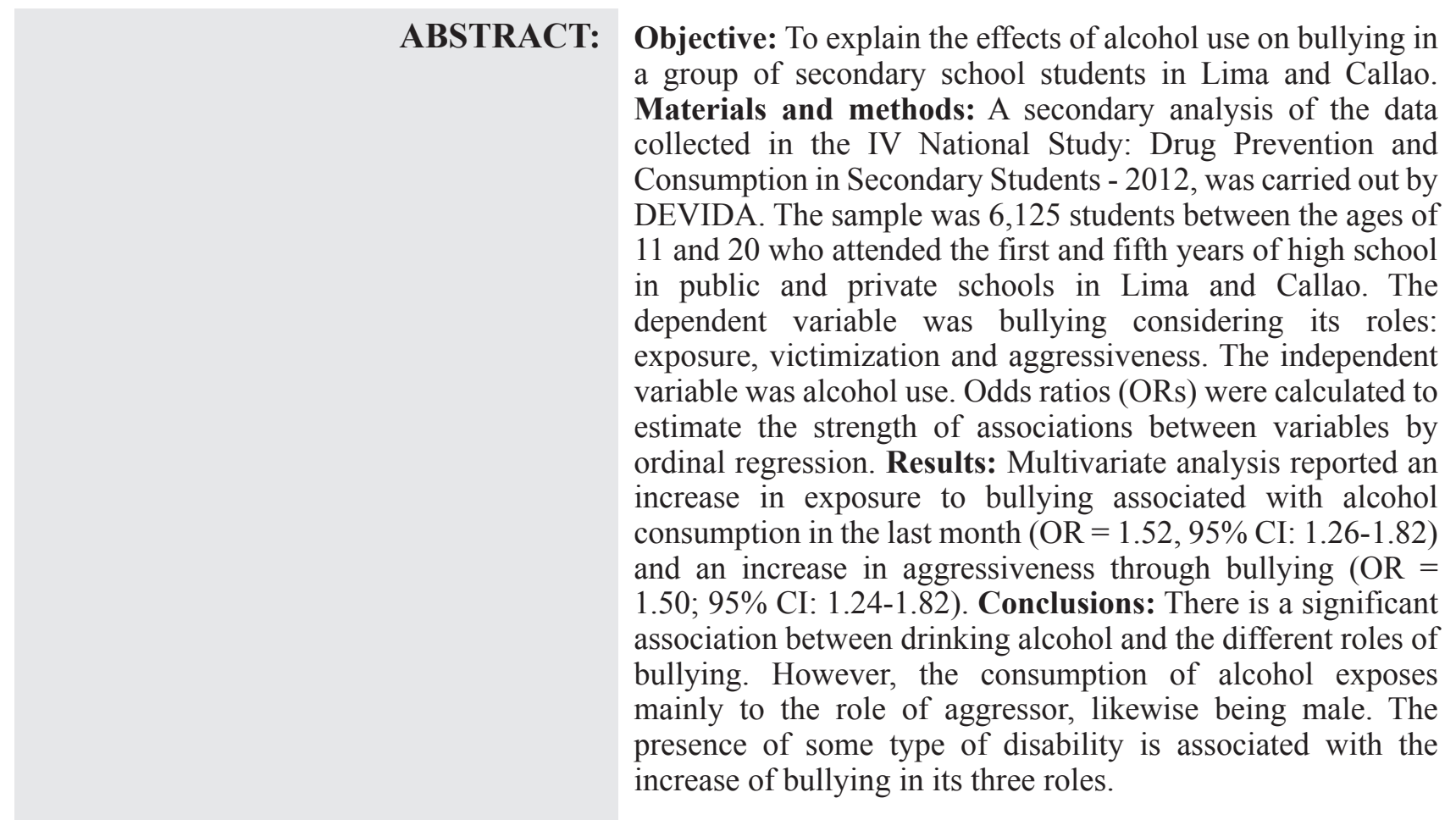

KEY WORDS: Underage Drinking; Bullying; Adolescent. 